\title{
Heavy-ion positrons explained
}

\section{The surprising observation of mono-energetic positrons in heavy-ion collisions may not be the signature of a novel particle but merely a sign that classical physics has been overlooked.}

THAT apparently small phenomena perceptively observed can lead to huge conceptual changes is a large part of the excitement of science. Newton's apocryphal falling apple is one example. Another is the recognition, by Meitner and Frisch (Nature 143, 239; 1939) that the presence of barium in the products of the reaction of neutrons with uranium must mean something odd, such as the fission of heavy nuclei.

So it is inevitable that there should have been suppressed excitement for well over a year about an unexpected phenomenon associated with the collision of heavy ions which appears first to have been recognized at the heavy-ion accelerator at Darmstadt, the West German town just south of Frankfurt which, since last weekend, will briefly be best known as the site from which the European Space Agency coordinated the flight of the Giotto spacecraft to Halley's comet.

The circumstances are these. The Darmstadt laboratory, in German the Gesellschaft für Schwerionenforschung, has a longstanding (and successful) interest in the creation of artificial heavy nuclei, to which end it is equipped to collide heavy ions with each other so energetically that the Coulomb repulsion of their positive charges are overcome. The surprise, first reported in 1983 , is that if the combined charge of the colliding ions is great enough (more than 163 units) the collisions are accompanied by the production of positrons with a precise energy (measured in the rest frame of the colliding ions).

Specifically, the energy distribution of the positrons appears to have a peak at an energy of $336 \mathrm{keV}$, which is itself apparently independent of the nature of the colliding ions. The width of the distribution is $75 \mathrm{keV}$ or so, implying that the positrons are produced from a source whose lifetime is long by the yardstick of the time taken by the nuclear collision of the ions, estimated at $10^{-21}$ seconds or so.

The excitement generated by these observations is easily imagined. The temptation has been irresistible to speculate about the properties of the unknown particles that are supposedly formed in the collision of heavy nuclei and which, by their radioactive decay, yield the positrons that are observed. One of the favourite candidates has been the axion, the particle that should exist if parity and isotopic spin are not conserved in weak nuclear reactions (see Nature 319, 717; 1986). There has even been the beginnings of a controversy on the subject, with some people saying that axions are produced because of their coupling to electrons, while others have held that the production process involves the interaction of hadronic particles, nuclear matter proper.

It now seems more probable that both camps are wrong, and that the unexpected observation of positrons in heavy-ion collisions is not so much evidence of a novel state of matter but rather a sign that even the people who should know much better have overlooked what should now be elementary physics. That, at least, is the implication of a modest article by CheukYin Wong, from the Oak Ridge National Laboratory in the United States, in the 10 March issue of Physical Review Letters (56, 1047; 1986).

Wong's argument is essentially quite simple. It turns on what should be by now Dirac's familiar description of what the vacuum is like. The point is merely that when Dirac first recognized more than half a century ago that a properly relativistic equation for the quantum motion of a free electron would admit of energies that are negative as well as positive, he was compelled to save the appearances by supposing that all the negative energy levels were in the real world completely occupied. If this were not the case, ordinary free electrons would forever be making the transition from a positive to a negative energy level, with the result that we would live in a world filled with radiation more energetic than the equivalent of $500 \mathrm{keV}$, which is half the smallest gap between a positive and negative electron energy level.

As the world knows, Dirac's view, strange though it may have seemed at the time, has been a great success. A positron is nothing but a hole in the sea of ordinary electrons with negative energy. The annihilation of positrons by ordinary electrons is the occupation of such a hole by an ordinary electron. The creation of an electron pair by a sufficiently energetic photon (whose energy must exceed the equivalent of twice $500 \mathrm{keV}$ ) consists simply of the promotion of an electron from the negative energy sea, leaving a hole that seems to be a positron, into an ordinary state of positive energy. Particle field theory is full of many more sophisticated applications of the same principle, under the banner of the "polarization of the vacuum" by suffi- ciently strong fields of force. (Near a black hole, gravitational forces produce other particles than electrons in this way.)

In essence, what Wong now says is that the positrons observed in heavy-ion collisions arise in much the same way. Dirac's concept of a sea of electrons is valid only in the absence of electrical or magnetic forces, and must therefore be modified in the neighbourhood of a nucleus. Wong points out that the energy of the two electrons in the lowest bound state in an atom whose atomic number is greater than 150 is negative, which is another way of saying (a little crudely) that its binding energy to the nucleus of the atom is greater than its rest energy, that due to its mass.

Indeed, Wong argues that for a sufficiently heavy ion entirely devoid of electrons, it would be energetically favourable that there should be created two electronpositron pairs, each of them crammed into the ground state of the nucleus (which would not be forbidden by Pauli's exclusion principle).

The extension of the argument to heavy-ion collisions is straightforward. Electron-positron pairs created in this way in the ground states of colliding ions would be shaken loose during the collision and thus left to their own devices. In the centre of mass of the system, they would be virtually at rest. Only mutual annihilation would be feasible. So why should not the electrons and positrons annihilate each other in pairs? Sometimes, no doubt, they do, producing $500-\mathrm{keV}$ radiation that will promptly be lost in the background. The more noticeable process, according to Wong, is that a single pair should annihilate each other, producing a single photon and transferring the excess momentum to a second positron that happens to be in the neighbourhood.

The surprise in this simple calculation is that the numbers turn out to be just right. The calculated kinetic energy of the surviving positron is $341 \mathrm{keV}$. Wong predicts that the accompanying photons (which might be detected by a coincidence experiment) should have an energy equivalent to $681 \mathrm{keV}$. There should also, he says, be observed the products of the inverse process in which excess momentum is transferred to an ordinary electron. That at least is something for which the experimentalists can hope to look, while they berate themselves (not to mention their theorist advisers) for having forgotten about Dirac.
John Maddox 